\title{
THE REFLECTION OF AN ELECTROMAGNETIC PLANE WAVE BY AN INFINITE SET OF PLATES, II*
}

\author{
BY \\ ALBERT E. HEINS ${ }^{1}$ AND J. F. CARLSON ${ }^{2}$ \\ Radiation Laboratory, ${ }^{3}$ Massachusetts Institute of Technology
}

1. Introduction. In Part $\mathrm{I}^{4}$ we have calculated rigorously with Fourier transform methods, the reflection and transmission coefficients due to the incidence of a plane electromagnetic wave on an infinite set of parallel staggered plates. We discussed there the case in which there was only one component of the electric field excited; that is, the incident electric field was parallel to the edges of the plates. We shall now discuss the same geometric structure when it is excited by a plane wave which has only a single component of the magnetic field which is parallel to the edges of the plates. In this case we shall see that the magnitude of the reflection and transmission coefficients are independent of the wavelength and depend only on the angle of stagger $\alpha$, and the direction of incidence of the plane wave $\theta$. We shall use the Fourier transform technique again, and since many of the calculations are parallel to those which we did in Part I, we shall only outline the procedure.

2. Formulation of the problem. We treat here the following problem. A plane monochromatic electromagnetic wave whose direction of propagation lies in the plane

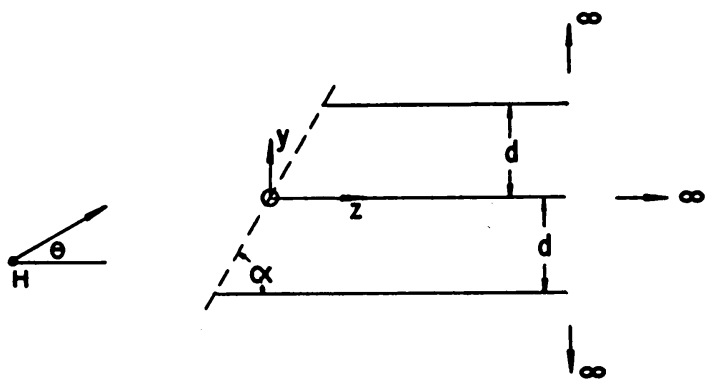

FIG. 1 of the paper, is incident upon an infinite set of staggered, equally spaced, semi-infinite metallic plates of zero thickness and perfect conductivity. These plates extend indefinitely in a direction perpendicular to the plane of the paper. (See Fig. 1 for a side view.) The angle of stagger with respect to a fixed direction (that of the cross section of the plates in Fig. 1) is $\alpha$, while the direction of propagation with respect to this fixed direc-

tion is $\theta$, where $\alpha-\pi<\theta<\alpha$ and $\theta<\alpha \leqq \pi / 2$.

We assume here that the incident wave has only one component of the magnetic field, that is, the component which is perpendicular to the plane of the paper. For such an excitation, no other components of the magnetic field can be excited. Thus; all components of the electric field can be derived from a single component of the magnetic field $H_{x}(y, z)=\psi(y, z)$. For this case, the components of the electric field lie in

* Received April 3, 1946.

${ }^{1}$ Now at the Carnegie Institute of Technology, Pittsburgh, Pa.

2 Now at Iowa State College, Ames, Iowa.

${ }^{3}$ This paper is based on work done for the Office of Scientific Research and Development under Contract OEMsr-262 with the Massachusetts Institute of Technology.

${ }^{4} \mathrm{~J}$. F. Carlson and A. E. Heins, The reflection of an electromagnetic plane wave by an infinite set of plates, I, this Quarterly, 4, 313-329 (1947). Hereafter we shall refer to this paper as I. 
the plane of the paper and we shall refer to this problem as an "E plane" problem.

Let us assume as in I, that the time dependence of all field quantities is $e^{-i k c t}$ so that Maxwell's equations may be written in spatial form as

and

$$
\nabla \times \mathbf{E}=i k \mathbf{H}
$$

$$
\nabla \times \mathbf{H}=-i k \mathbf{E},
$$

where $k=2 \pi / \lambda$, and $\lambda$ is the free space wave-length. We then have that $i k E_{\nu}=-\partial \psi / \partial z$ and $i k E_{z}=\partial \psi / \partial y$. Upon eliminating $E_{y}$ and $E_{z}$ from the above equations we get the two dimensional wave equation

$$
\frac{\partial^{2} \psi}{\partial y^{2}}+\frac{\partial^{2} \psi}{\partial z^{2}}+k^{2} \psi=0
$$

which is to be solved subject to the boundary condition $\partial \psi / \partial y=0$ on the metal plates, since $E_{z}$ is the tangential component of the electric field on these plates. There are also conditions at infinity on the function $\psi(y, z)$ which are similar to those which we required for $\phi(x, z)$ in $\mathrm{I}$.

We now formulate the equation which expresses the $z$ component of the electric field at any point $(y, z)$ in terms of the surface current density on the plates. Following a procedure similar to that developed in I, we find

$\psi(y, z)=\psi_{\mathrm{in}}{ }^{\mathrm{c}}(y, z)-\frac{i}{4} \sum_{m=-\infty}^{\infty} \int_{m \sigma}^{\infty} I_{m}\left(z^{\prime}\right) \frac{\partial H_{0}^{(1)}}{\partial y^{\prime}}\left[k \sqrt{\left(z-z^{\prime}\right)^{2}+\left(y+y^{\prime}\right)^{2}}\right] d z^{\prime}$

where $y^{\prime}=m d, g=d \cot \alpha$ and $\psi_{\mathrm{ino}}(y, z)=e^{i k(y \sin \theta+z \cos \theta)}$ is the incident magnetic field. $H_{0}^{(1)}\left[k \sqrt{\left(x-z^{\prime}\right)^{2}+\left(y-y^{\prime}\right)^{2}}\right]$ is the free space Green's function which we have described in $I$. Due to the form in which $y$ and $y^{\prime}$ appear, we may write (2.1) as

$$
\psi(y, z)=\psi_{\mathrm{ino}}(y, z)+\frac{i}{4} \frac{\partial}{\partial y} \sum_{m=-\infty}^{\infty} \int_{m g}^{\infty} I_{m}\left(z^{\prime}\right) H_{0}^{(1)}\left[k \sqrt{\left(z-z^{\prime}\right)^{2}+(y-m \bar{d})^{2}}\right] d z^{\prime} .
$$

Now $\partial \psi / \partial y$, which is proportional to $E_{z}(y, z)$, is given by the equation

$$
\begin{aligned}
& \frac{\partial}{\partial y} \psi(y, z)=\frac{\partial}{\partial y} \psi_{\mathrm{inc}}(y, z) \\
& \quad+\frac{i}{4} \frac{\partial^{2}}{\partial y^{2}} \sum_{m=-\infty}^{\infty} \int_{m_{\vartheta}} I_{m}\left(z^{\prime}\right) H_{0}^{(1)}\left[k \sqrt{\left(z-z^{\prime}\right)^{2}+(y-m d)^{2}}\right] d z^{\prime},
\end{aligned}
$$

and since $E_{z}(y, z)$ vanishes for $y=n d, z>n d \cot \alpha, n=0, \pm 1, \ldots$ Eq. (2.2) leads to an infinite set of inhomogeneous integral equations of the Wiener-Hopf type similar to those developed in I.

Some remarks are now in order about the range of values of $d / \lambda$ which we will assume here. In the first place, we suppose that in the parallel plate regions, $\psi(y, z)$ is proportional to $e^{i k z}$ for $z$ sufficiently large and positive. That is, the parallel plate region can sustain the so-called principal mode, a mode which cannot occur in the $H$ plane case. In order that no higher mode propagate, we must now have that $0<d / \lambda<\frac{1}{2}$. We further assume that there is only a single reflected wave. This assumption puts further limitations on $d / \lambda$ as well as on $\theta$, and we shall discuss them when we have obtained the final solution of the problem. 
3. Fourier transform solution of the integral equation. We have already shown in I, Section 3, that the surface current density on any plate can be expressed in terms of the surface current density of a given plate, the argument being that we had a geometric structure with two types of periodicities. The same argument shows us now that

$$
I_{m}(z-m g)=I_{0}(z) e^{i k m(g \cos \theta+d \sin \theta)},
$$

where $I_{0}(z)$ is the surface current density on the zeroth plate. With this reduction and an appropriate change of variables, the integral equation which we have to solve, now reads

$$
\begin{aligned}
0= & \frac{\partial}{\partial y} \psi_{\text {inc }}(y, z) \\
& +\frac{i}{4} \sum_{m=-\infty}^{\infty} \int_{0}^{\infty} I_{0}\left(z^{\prime}\right) e^{i k \mu m} \frac{\partial^{2}}{\partial y^{2}} H_{0}^{(1)}\left[k \sqrt{\left\{z-z^{\prime}+(n-m) g\right\}^{2}+(y-m d)^{2}}\right] d z^{\prime} z>0,
\end{aligned}
$$

where now $g=d \cot \alpha$, and $\mu=g \cos \theta+d \sin \theta$. The $y$ derivatives in (3.1) are understood to be evaluated at $y=n d$. Equation (3.1) is an inhomogeneous Wiener-Hopf integral equation, which may be rewritten as

$0=i k \sin \theta e^{i k z \cos \theta}$

$$
+\frac{i}{4} \sum_{p=-\infty}^{\infty} \int_{0}^{\infty} I_{0}\left(z^{\prime}\right) e^{i k \mu p} \frac{\partial^{2}}{\partial y^{2}} H_{0}^{(1)}\left[k \sqrt{\left(z-z^{\prime}+p g\right)^{2}+(y-n d-p \dot{d})^{2}}\right] d z^{\prime} z>0 .
$$

The dependence of Eq. (3.2) on $n$ is not explicit as the differentiation of $H_{0}^{(1)}\left[k \sqrt{\left(z-z^{\prime}+p g\right)^{2}+(y-n d-p d)^{2}}\right]$ with respect to $y$ will demonstrate. We now extend Eq. (3.2) to hold for all $z$ by writing it as

$$
\begin{aligned}
F(z)= & \left.\frac{\partial}{\partial y} \psi_{\mathrm{inc}}(y, z)\right|_{y=0} \\
& +\frac{i}{4} \sum_{p=-\infty}^{\infty} \int_{-\infty}^{\infty} I_{0}\left(z^{\prime}\right) e^{i k \mu p} \frac{\partial^{2}}{\partial y^{2}} H_{0}^{(1)}\left[k \sqrt{\left(z-z^{\prime}+p g\right)^{2}+(y-n d-p d)^{2}} d z^{\prime},\right.
\end{aligned}
$$

where we define

$$
\begin{array}{r}
F(z) \equiv 0 \quad z>0, \quad I_{0}(z) \equiv 0 \quad z<0, \\
\left.\frac{\partial}{\partial y} \psi_{\mathrm{inc}}(y, z)\right|_{y=0} \begin{cases}\equiv i k \sin \theta e^{i k z \cos \theta} & z>0, \\
\equiv 0 & z<0 .\end{cases}
\end{array}
$$

We assume as in I, for analytical convenience, that $k$ has a small positive imaginary part.

By arguments which we employed in I, we can show that $F(z)$ is asymptotic to $e^{i k z \cos (2 \alpha-\theta)}$ for $z$ large and negative and therefore it has a Fourier transform

$$
\int_{-\infty}^{0} e^{-i w z^{\prime} F\left(z^{\prime}\right) d z^{\prime}}
$$

which is regular in the upper half of the $\omega$ plane $\Im m w>+\Im m k \cos (2 \alpha-\theta)$. Since $I_{0}(z)$ is asymptotic to $e^{i k z}$ for $z$ large and positive, that is, the parallel plate regions are of such spacing as to permit the propagation of the principal mode only, the Fourier 
transform of $I_{0}(z)$ is regular in the lower half of the $w$ plane $\Im$ mw $<\Im_{m k} .^{5}$ The transform of $\partial \psi_{\mathrm{inc}}(y, z) /\left.\partial y\right|_{y=0}$ is regular in the lower half of the $w$ plane, $\Im \mathfrak{m} w<\Im \mathfrak{m} k \cos \theta$. Finally, the transform of the kernel of Eq. (3.3) is

$$
\frac{\sqrt{k^{2}-w^{2}} \sin d \sqrt{k^{2}-w^{2}}}{\cos d \sqrt{k^{2}-w^{2}}-\cos (k \mu-w d)}=K(w)=\frac{K_{-}(w)}{K_{+}(w)}
$$

and it is regular in the strip

$$
\Im \mathfrak{m} k \cos (2 \alpha-\theta)<\Im \mathfrak{m} w<\Im \mathfrak{m} k \cos \theta .
$$

Since all of the transforms involved in Eq. (3.3) have a common strip of regularity in the $w$ plane, it is thus legitimate to apply the Fourier transform to this equation to get

$$
D(w)=\frac{k \sin \theta}{w-k \cos \theta}-\frac{\sqrt{k^{2}-w^{2}} \sin d \sqrt{k^{2}-w^{2}} J(w)}{2\left[\cos d \sqrt{k^{2}-w^{2}}-\cos (k \mu-w d)\right]},
$$

where $D(w)$ and $J(w)$ are the respective transforms of $F(z)$ and $I_{0}(z)$.

We are once again led to a problem in factorization of the coefficient of $J(w)$ into two functions, one of which is regular in the upper half plane $\Im \mathfrak{m} w>\Im \mathfrak{m} k \cos (2 \alpha-\theta)$, while the other is regular in the lower half plane $\Im \mathfrak{m} w<\Im \mathfrak{m} k \cos \theta$. The factorization of the denominator $\cos d \sqrt{k^{2}-w^{2}}-\cos (k \mu-w d)$ is the same as it was in I, save for the fact that $\rho$ and $a$ have been replaced by $\mu$ and $d$ respectively. The numerator $\sqrt{k^{2}-w^{2}} \sin d \sqrt{k^{2}-w^{2}}$ may be written in factor form as

$$
\begin{array}{r}
d\left(k^{2}-w^{2}\right) \prod_{n=1}^{\infty}\left[\sqrt{1-(k d / n \pi)^{2}}+(i w d / n \pi)\right] e^{-i w d / n \pi} \\
\cdot \prod_{n=1}^{\infty}\left[\sqrt{1-(k d / n \pi)^{2}}-(i w d / n \pi)\right] e^{i w d / n \pi} .
\end{array}
$$

Clearly $(k-w) \prod_{n=1}^{\infty}\left[\sqrt{1-(k d / n x)^{2}}+i w d / n x\right] e^{-i w d / n x}$ has no zeros in the lower half plane $\Im \mathfrak{m} w<\Im \mathfrak{m} k$, while the factor $(k+w) \prod_{n=1}^{\infty}\left[\sqrt{1-(k d / n x)^{2}}-(i w d / n x)\right] e^{i w d / n x}$ has no zeros in the upper half plane $\Im \mathfrak{m} w>\Im \mathfrak{m}(-k)$. Thus

$$
\frac{\sqrt{k^{2}-w^{2}} \sin d \sqrt{k^{2}-w^{2}}}{\cos d \sqrt{k^{2}-w^{2}}-\cos (k \mu-w d)}
$$

may be factored into two functions, $K_{+}(w)$ and $K_{-}(w)$, where $K_{+}(w)$ is regular in the upper half plane $\Im \mathfrak{m} w>\Im \mathfrak{m} k \cos (2 \alpha-\theta)$, while $K_{-}(w)$ is regular in the lower half plane $\Im_{\mathfrak{m}}<\mathfrak{I} \mathfrak{m} k \cos \theta$. We have

$$
\begin{aligned}
& K_{-}(w)=\left[(k-w) \prod_{n=1}^{\infty}\left[\sqrt{1-(k d / n \pi)^{2}}+(i w d / n \pi)\right] e^{-i w d / n \pi} e^{\chi(w)}\right] \\
& \cdot\left[\left(w-\sigma_{1}\right) \prod_{n=-\infty}^{-1}\left[\Delta_{n}-i \Psi_{n}\right] e^{(k \mu-w g+i w d) / 2 n \pi+i\left(\frac{1}{2} \pi-\alpha\right)} \prod_{n=1}^{\infty}\left[\Delta_{n}+i \Psi_{n}\right] e^{(k \mu-w g+i w d) / 2 n \pi-i\left(\frac{1}{2} \pi-\alpha\right)}\right]
\end{aligned}
$$

5 It has been tacitly assumed that $F(z)$ and $I_{0}(z)$ are integrable for all finite $z$. This we can show directly from the integral equation. 
and

$$
\begin{aligned}
& \frac{1}{K_{+}(w)}=\frac{2 d}{\left(d^{2}+g^{2}\right)}\left[(k+w) \prod_{n=1}^{\infty}\left[\sqrt{1-(k d / n \pi)^{2}}-(i w d / n \pi)\right] e^{i w d / n \pi} e^{-\chi(w)}\right] \\
& \cdot\left[\left(w-\sigma_{2}\right) \prod_{n=-\infty}^{-1}\left[\Delta_{n}+i \Psi_{n}\right] e^{(k \mu-w g+i w d) / 2 n \pi-i\left(\frac{1}{2} \pi-\alpha\right)} \prod_{n=1}^{\infty}\left[\Delta_{n}-i \Psi_{n}\right] e^{(k \mu-w g+i w d) / 2 n \pi+i\left(\frac{1}{1} \pi-\alpha\right)}\right]
\end{aligned}
$$

The $\Delta_{n}$ and $\Psi_{n}$ are the same functions of $w$ which we encountered in I save for the interchange of $\rho$ and $a$, by $\mu$ and $d$ respectively; $\sigma_{1}=k \cos \theta, \sigma_{2}=k \cos (2 \alpha-\theta)$ while $\chi(w)$ is an integral function introduced into the decomposition of $K(w)$ to render the portions $K_{+}(w)$ and $K_{-}(w)$ algebraic in growth in their respective half planes of regularity as $|w|$ becomes infinite.

The decomposition of Eq. (3.4) into two functions, one of which is regular in the upper half plane $\Im \mathfrak{m} w>\Im \mathfrak{m} k \cos (2 \alpha-\theta)$ and the other of which is regular in the lower half plane $\Im m w<\Im m k \cos \theta$ follows as before. We now have

$$
\begin{aligned}
D(w) K_{+}(w)-\frac{k \sin \theta\left[K_{+}(w)-K_{+}(k \cos \theta)\right]}{w-k \cos \theta} & \\
& =-\frac{K_{-}(w)}{2} J(w)+\frac{k K_{+}(k \cos \theta) \sin \theta}{w-k \cos \theta} .
\end{aligned}
$$

Since the left side of (3.4) is regular in the lower half plane $\Im \mathfrak{m} w<\Im \mathfrak{m} k \cos \theta$ and the right side is regular in the upper half plane $\Im \mathfrak{m} w>\Im \mathfrak{m} k \cos (2 \alpha-\theta)$, while both sides are regular in the strip $\Im \mathrm{m} k \cos (2 \alpha-\theta)<\Im m w<k \cos \theta$, it follows that both sides of Eq. (3.5) are equal to an integral function. The final decomposition then gives us

$$
\begin{aligned}
D(w) K_{+}(w)-\frac{k \sin \theta\left[K_{+}(w)-K_{+}(k \cos \theta)\right]}{w-k \cos \theta} & =\text { Integral function, } \\
-\frac{1}{2} J(w) K_{-}(w)+\frac{k K_{+}(k \cos \theta) \sin \theta}{w-k \cos \theta} & =\text { Integral function. }
\end{aligned}
$$

We now turn to the evaluation of the integral function of separation and $\chi(w)$. The functional form of $\chi(w)$ follows from the asymptotic form of $K_{-}(w),|w| \rightarrow \infty$, $\Im \mathfrak{m} w<0$ or of $K_{+}(w),|w| \rightarrow \infty, \Im \mathfrak{m} w>0$. The method of calculating these asymptotic forms is the same as in I. We find now that

$$
K_{-}(w) \sim w^{-1 / 2}, \quad \Im m w<0 \quad|w| \rightarrow \infty,
$$

provided $\chi(w)$ is chosen as $-(i w d / \pi)[(\alpha-\pi / 2 \cot \alpha)-\ln 2 \sin \alpha]$. If we let $|w| \rightarrow \infty$, $\Im \mathfrak{m} w<0$, it is clear that the integral function of separation in Eq. (3.7) approaches zero in the lower half plane. A similar calculation for Eq. (3.6) shows that the integral function approaches zero in the upper half plane $|w| \rightarrow \infty, \Im m w>0$. Since this integral function is everywhere bounded and approaches zero for $|w| \rightarrow \infty$, it is identically zero. From (3.7) we then get the Fourier transform of $I_{0}(z)$

$$
J(w)=\frac{2 k K_{+}(k \cos \theta) \sin \theta}{K_{-}(w)[w-k \cos \theta]} .
$$

4. Investigation of the far fields. In order to find the amplitudes of the reflected 
and transmitted waves we must find the asymptotic form of $\psi(y, z)$ as $|z| \rightarrow \infty$. This can be done by noting that.Eq. (2.1) may be written in Fourier integral representation as

$$
\begin{aligned}
\psi(x, z)= & \psi_{\mathrm{inc}}(y, z)-\frac{1}{4 x} \int_{C} d w J(w) e^{i w z+i(k \mu-w g) n} \\
& \frac{\cos (y-d n-d) \sqrt{k^{2}-w^{2}}-e^{i(k \mu-w g)} \cos (d n-y) \sqrt{k^{2}-w^{2}}}{\cos \sqrt{k^{2}-w^{2}} d-\cos (k \mu-w g)}
\end{aligned}
$$

where the contour $C$ is a path in the strip of regularity $\Im \mathfrak{m} k \cos (2 \alpha-\theta)<\Im \mathfrak{m} w$ $<\Im \mathfrak{m} k \cos \theta$ and is closed above or below by a semi-circle which passes between the poles of the integrand, depending on whether $z$ is greater or less than zero. The integration along the semi-circular arc gives no contribution to the integral (4.1) when its radius becomes infinite. $n$ is the largest integer in $y / d$.

Let us first consider the asymptotic form of $\psi(y, z)$ as $z$ becomes large and positive. In the integral in Eq. (4.1), we see that the two poles which give propagating mode contributions are at $w=\sigma_{1}=k \cos \theta$, and $w=k$. All other poles give exponential factors in $z$ which attenuate for $z$ large and positive. The contour $C$ is, of course, closed in the upper half plane. The integral in (4.1) is then

$$
\begin{aligned}
& -\frac{1}{2 \pi} \int_{C} d w \frac{k K_{+}(k \cos \theta) \sin \theta e^{i w z} e^{i(k \mu-w g) n}}{(w-k \cos \theta) \sqrt{k^{2}-w^{2}} K_{+}(w) \sin d \sqrt{k^{2}-w^{2}}} \\
\cdot & {\left[\cos (y-d n-d) \sqrt{k^{2}-w^{2}}-e^{i(k \mu-w g)} \cos (d n-y) \sqrt{k^{2}}-w^{2}\right] } \\
= & -e^{i k(z \cos \theta+y \sin \theta)} \\
& -\frac{i k \sin \theta K_{+}(k \cos \theta) e^{i k z+i k n d \sin \theta}}{2 k d(1-\cos \theta) K_{+}(k)}\left(1-e^{i(k \mu-k g)}\right) \\
& + \text { exponential terms in } y \text { and } z \text { which are attenuated for } z \text { large and positive. }
\end{aligned}
$$

Thus, for $z$ large and positive, $\psi(y, z)$ is asymptotic to

$$
\frac{-i \sin \theta K_{+}(k \cos \theta) e^{i k n d \sin \theta+i k z}\left(i-e^{i k d \sin \theta}\right)}{2 k d K_{+}(k)(1-\cos \theta)},
$$

that is, the correct lowest mode functional form in the parallel plate region for " $E$ plane" polarization. The magnitude $|T|$ of the transmission coefficient is then

$$
|T|=\frac{\sin \theta\left|K_{+}(k \cos \theta) \sin [k(\mu-g) / 2]\right|}{k d(1-\cos \theta)\left|K_{+}(k)\right|},
$$

which reduces to

$$
|T|=\frac{\sin (\alpha-\theta)}{\sin \left(\alpha-\frac{1}{2} \theta\right) \cos \frac{1}{2} \theta}
$$

provided now $k$ is taken to be purely real.

We now compute the amplitude of the reflected wave by the same method that we employed in I. That is, for $y$ fixed and $z$ large and negative, we close the path by a large semi-circular arc in the lower half plane which passes between the poles in this half plane. If we allow the radius of this semi-circle to become infinite, the usual 
arguments show that there is no contribution to the integral from this circular arc. The residue calculation then gives us for $y$ fixed, $z$ large and negative,

$$
\begin{aligned}
\psi(y, z)=\psi_{\text {inc }}(y, z) & +\frac{e^{i k[y \sin (2 \alpha-\theta)+z \cos (2 \alpha-0)]} K_{+}(k \cos \theta) \sin \theta}{2 k K_{+}^{\prime}[k \cos (2 \alpha-\theta)] \sin \alpha \sin (2 \alpha-\theta) \sin (\alpha-\theta)} \\
& + \text { terms which attenuate exponentially for } z \text { large and negative. }
\end{aligned}
$$

With the amplitude of the incident wave taken as unity, the amplitude of the reflected wave, that is, the reflection coefficient is

$$
R=\frac{K_{+}(k \cos \theta) \sin \theta}{2 k K_{+}^{\prime}[k \cos (2 \alpha-\theta)] \sin \alpha \sin (2 \alpha-\theta) \sin (\alpha-\theta)}
$$

which simplifies to

$$
R=\tan \frac{1}{2} \theta \cot \left(\alpha-\frac{1}{2} \theta\right) e^{i \Phi}
$$

when $k$ is taken real. $\Phi$ is the phase angle of the reflection coefficient and is of the same functional form as the $\Theta_{1}^{\prime}-\Theta_{2}^{\prime}$ found in I, Section 5, save now for the fact that $a$ has been replaced by $d$. We have finally

$$
|R|=\tan \frac{1}{2} \theta \cot \left(\alpha-\frac{1}{2} \theta\right) \text {. }
$$

Conservation of power flow from free space to any parallel plate region gives the following relation between $|R|$ and $|T|$ :

$$
\frac{\sin (\alpha-\theta)}{\sin \alpha}\left[1-|R|^{2}\right]=|T|^{2} .
$$

The values of $|R|$ and $|T|$ clearly satisfy this condition. Finally, we note that the condition for a single reflected wave is the same as it was in I, namely

$$
\frac{2 d}{\lambda}<\frac{\sin \alpha}{\cos ^{2}[(\theta-\alpha) / 2]} .
$$

Since $0<d / \lambda<\frac{1}{2}$, the inequality (4.3) is not as severe as the comparable relation in I.

It is illuminating to introduce an angle $i$ into the formulas (4.2) and (4.3). This angle $i$ is the angle which the direction of propagation of the incident wave makes with the normal to the trace of the edges of the parallel plates and is equal to $\frac{1}{2} \pi-\alpha+\theta$. The magnitude of the reflection coefficient then becomes

$$
|R|=\frac{\sin \alpha-\cos i}{\sin \alpha+\cos i}
$$

while the magnitude of the transmission coefficient becomes

$$
|T|=\frac{2 \cos i}{\sin \alpha+\cos i}
$$

There will be no reflection from this structure if $|R|=0$ or $\alpha=\frac{1}{2} \pi-i$, that is, the direction of propagation of the incident wave is parallel to surfaces of the parallel plates. In this case, of course, $|T|$ is unity. 